\title{
MEDICINE
}

\section{DIAGNOSTIC VALUE OF SYSTEMIC LUPUS ERYTHEMATOSUS CLASSIFICATION CRITERIA (AMERICAN COLLEGE OF RHEUMATOLOGY, 1997)}

\author{
U. Abrahamovych ${ }^{1}$, O. Abrahamovych $^{1}$, O. Nadashkevych $^{1}$, A. Svintsitskyi ${ }^{2}$, O. Synenkyi $^{3}$ \\ ${ }^{1}$ Danylo Halytsky Lviv National Medical University \\ ${ }^{2}$ Bogomolets National Medical University \\ ${ }^{3}$ Communal Non-profit Institution of Lviv Regional Council "Lviv Regional Clinical Hospital"
}

DOI: https://doi.org/10.31435/rsglobal_wos/30042020/7041

\section{ARTICLE INFO}

Received: 12 February 2020

Accepted: 04 April 2020

Published: 30 April 2020

\section{KEYWORDS}

systemic lupus erythematosus, American College of Rheumatology criteria,

diagnosis,

sensitivity,

specificity,

accuracy

\begin{abstract}
The criteria for the classification of systemic lupus erythematosus were proposed in 1971 by the American College of Rheumatology. They have been clarified since then, but need to be revised.

Objective. To determine the diagnostic value of the criteria for the classification of systemic lupus erythematosus proposed by the American College of Rheumatology.

Materials and methods. 370 patients (331 women (89.46\%) and 39 men (10.54\%), average age $41.24 \pm 0.63$ years) with SLE and 234 patients (150 women $(64.10 \%)$ and 84 men $(35.90 \%)$, average age $48.82 \pm 0.85$ years) with other rheumatic diseases (rheumatoid arthritis, psoriatic arthritis, ankylosing spondylitis) were randomly enrolled into the study. The patients had undergone comprehensive clinical-laboratory and instrumental examinations in 2010-2018 before they received treatment. The analysis was conducted in MS Excel and SPSS by constructing contingency tables and calculating indicators of diagnostic value.

Results. We identified the following criteria as those that can with statistical significance predict the presence of systemic lupus erythematosus: butterfly rash, photosensitivity, serositis (pleuritis, pericarditis), neurologic disorders (seizures, psychosis), thrombocytopenia, renal disorders (proteinuria, cylindruria), anti-dsDNA and antinuclear antibodies.
\end{abstract}

Citation: U. Abrahamovych, O. Abrahamovych, O. Nadashkevych, A. Svintsitskyi, O. Synenkyi. (2020) Diagnostic Value of Systemic Lupus Erythematosus Classification Criteria (American College of Rheumatology, 1997). International Academy Journal Web of Scholar. 4(46). doi: 10.31435/rsglobal_wos/30042020/7041

Copyright: (C 2020 U. Abrahamovych, O. Abrahamovych, O. Nadashkevych, A. Svintsitskyi, O. Synenkyi. This is an open-access article distributed under the terms of the Creative Commons Attribution License (CC BY). The use, distribution or reproduction in other forums is permitted, provided the original author(s) or licensor are credited and that the original publication in this journal is cited, in accordance with accepted academic practice. No use, distribution or reproduction is permitted which does not comply with these terms.

Topicality. The criteria for the classification of systemic lupus erythematosus (SLE) were first proposed in 1971 by the American College of Rheumatology (ACR) [2, 3]. Although they have been revised several times since then (1982 [5], 1997 [4]), according to literature and our own clinical experience, there are often situations where the diagnosis of the disease is obvious, but the number of met criteria is not sufficient and vice versa. Such situations have prompted this study.

Objective. To determine the diagnostic value of systemic lupus erythematosus classification criteria proposed by the American College of Rheumatology (1997).

Materials and methods. Having obtained written consents to participate in comprehensive examinations in accordance with the principles of Helsinki Human Rights Declaration, Council of Europe Convention on Human Rights and Biomedicine, the relevant laws of Ukraine and other international instruments, after stratification by the presence of SLE and other rheumatic diseases 
(rheumatoid arthritis, psoriatic arthritis, ankylosing spondylitis), we randomly enrolled 370 patients with SLE (hereinafter referred to as „SLE group”; 331 women (89.46\%) and 39 men (10.54\%), aged 18 to 74 years) and 234 patients with other rheumatic diseases (hereinafter referred to as „,comparison group" (CG), 150 women (64.10\%) and 84 men (35.90\%), aged 19 to 84 years) into the study. All patients had undergone comprehensive clinical-laboratory and instrumental examinations of all organs and systems before they received treatment in 2010-2018 at the Rheumatology Department of Lviv Regional Clinical Hospital, the clinical base of the Departments of Internal Medicine No. 1 and No. 2 of Danylo Halytsky National Medical University. The treatment was administered in accordance with the Order of the Ministry for Health of Ukraine No. 676 of October 12, 2006 "On the Approval of Protocols for the Provision of Medical Care under the Rheumatology Specialty". In addition to routine examinations, all patients underwent tests for anti-double stranded DNA (anti-dsDNA) antibodies, antiphospholipid antibodies (IgG and $\operatorname{IgM}$ ), lupus anticoagulants, antinuclear antibodies (ANAs).

The study was based on the statistical analysis with the calculation of the odds of establishing a correct diagnosis of SLE. It was conducted in two consecutive steps. The first step involved assessing the diagnostic value of the ACR criteria, and the second step involved assessing the diagnostic value of their individual components.

The reliable probability of diagnosis was determined using indicators of diagnostic value: sensitivity, specificity, and accuracy. Sensitivity (true positive rate) is an indicator of medical statistics that denotes the proportion of positive results correctly identifying the disease. Specificity (true negative rate) is an indicator that denotes the proportion of negative results correctly identifying healthy people (as not having that disease). Accuracy is a share of correct assessments (diagnoses) made on the basis of information about positive or negative results [1]. The odds ratio and the coefficient of association (contingency) were also determined using these indicators. The odds ratio is a statistic that shows how the absence or presence of a particular result was associated with the presence or absence of a particular disease in a group. The coefficient of association (contingency) is a statistic that denotes the strength of the stochastic relationship between qualitative manifestations alternative random variables [1].

The actual data was processed on a personal computer with MS Excel and SPSS by constructing contingency tables and calculating indicators of diagnostic value. The relationship between SLE and the indicator in question was considered to be statistically significant if the absolute number of association coefficient exceeded 0.5 (or if the absolute number of contingency coefficient exceeded 0.3).

Results and discussion. The results of the first step are shown in Table 1 and Figure 1. The purpose of the first step was to determine the diagnostic value of the ACR criteria by conducting statistical analysis and calculating the odds of making the correct diagnosis (SLE).

Table 1. Diagnostic Value of Systemic Lupus Erythematosus Classification Criteria as Proposed by the American College of Rheumatology in 1997

\begin{tabular}{|c|c|c|c|c|c|c|c|}
\hline No. & Diagnostic criteria & Sensitivity, $\%$ & Specificity, $\%$ & Accuracy, $\%$ & Odds ratio & $\begin{array}{c}\text { Coefficient } \\
\text { of } \\
\text { association }\end{array}$ & $\begin{array}{c}\text { Coefficient } \\
\text { of } \\
\text { contingency }\end{array}$ \\
\hline 1. & Malar rash & 54.32 & 100.00 & 72.02 & - & 1 & $0.56 *$ \\
\hline 2. & Discoid rash & 9.73 & 100.00 & 44.70 & - & 1 & 0.20 \\
\hline 3. & Photosensitivity & 34.32 & 100.00 & 59.77 & - & 1 & $0.41 *$ \\
\hline 4. & Oral ulcers & 18.85 & 100.00 & 05.05 & - & 1 & 0 \\
\hline 5. & Arthritis & 20.54 & 7.27 & 15.40 & 0.02 & -0.96 & - \\
\hline 6. & Serositis & 22.70 & 100.00 & 52.65 & - & 1 & $0.32 *$ \\
\hline 7. & Renal disorders & 48.38 & 86.48 & 62.67 & 6.00 & $0.71 *$ & - \\
\hline 8. & $\begin{array}{c}\text { Neurologic } \\
\text { disorders }\end{array}$ & 12.43 & 98.70 & 45.59 & 10.79 & $0.83 *$ & - \\
\hline 9. & $\begin{array}{c}\text { Hematologic } \\
\text { disorders }\end{array}$ & 45.68 & 52.56 & 48.34 & 0.93 & -0.04 & - \\
\hline 10. & $\begin{array}{c}\text { Immunologic } \\
\text { disorders }\end{array}$ & 88.30 & 96.55 & 89.98 & 211.4 & $0.99 *$ & - \\
\hline 11. & ANAs & 87.99 & 84.21 & 87.35 & 39.06 & $0.95 *$ & - \\
\hline
\end{tabular}

Note: $*-$ the presence of SLE is statistically significant, $p<0.05$. 


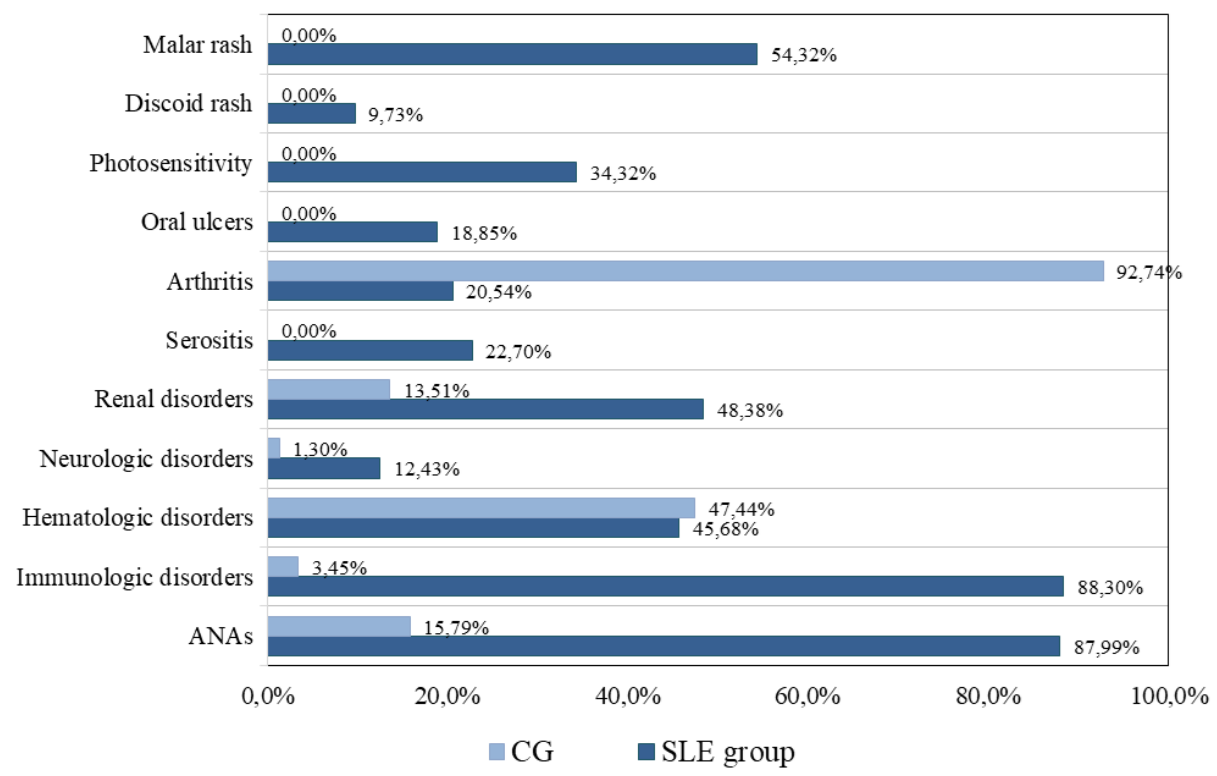

Fig.1. Prevalence of the ACR Criteria in Patients with SLE and in Patients with Other Rheumatic Diseases

The malar rash (butterfly rash) was detected in 201 patients (54.32\%) of the SLE group. No patients $(0.00 \%)$ of the comparison group had an erythematous rash on their faces. The sensitivity of the criterion was $54.32 \%$, specificity $-100.00 \%$, accuracy $-72.02 \%$. There was a statistically significant positive relationship between the criterion and the presence of SLE in patients (contingency coefficient of 0.56) - confirming the diagnostic value of the criterion.

The discoid rash was detected in only 36 patients $(9.73 \%)$ of the SLE group. It was not detected in any patient $(0.00 \%)$ of the comparison group. The sensitivity of the criterion was $9.73 \%$, specificity $-100.00 \%$, and accuracy $-44.70 \%$. There was no statistically significant relationship between the criterion and the presence of the disease (contingency coefficient of 0.20 ).

Photosensitivity - rash due to atypical reaction to sunlight - occurred in 127 patients $(34.32 \%)$ of the SLE group. It did not occur in any patient $(0.00 \%)$ of the comparison group. The sensitivity of the criterion was $34.32 \%$, specificity $-100.00 \%$, and accuracy $-56.77 \%$. There was no statistically significant relationship between the presence of photosensitivity and SLE (contingency coefficient of 0.41).

Oral ulcers were detected, due to objective examinations, in 69 patients $(18.85 \%)$ of the SLE group. There were no such lesions in patients $(0.00 \%)$ of the comparison group. The sensitivity of the criterion was $18.85 \%$, specificity was $100.00 \%$, and accuracy was $50.50 \%$. There was no significant relationship between the criterion and the presence of SLE (contingency coefficient of 0.29).

Arthritis was detected in 76 patients (20.54\%) of the SLE group and in 217 patients (92.74\%) of the comparison group. The sensitivity of the criterion for the diagnosis of SLE was $20.54 \%$, specificity was $7.27 \%$, and accuracy was $15.40 \%$. There was a statistically significant negative relationship between the presence of arthritis and SLE (association coefficient of -0.96). Consequently, the presence of arthritis significantly rules out SLE.

Serositis, including the presence of pericarditis and/or pleuritis, was detected in 84 patients (22.70\%) of the SLE group. It was not detected in any patient $(0.00 \%)$ of the comparison group. The sensitivity of the criterion was $22.70 \%$, specificity $-100.00 \%$, accuracy $-52.65 \%$. There was a statistically significant relationship between the presence of serositis and SLE (contingency coefficient of -0.32).

Renal disorders - proteinuria or cylindruria - were detected in 179 patients $(48.38 \%)$ of the SLE group and in 30 patients $(13.51 \%)$ of the comparison group. The sensitivity of the criterion was $48.38 \%$, specificity $-86.49 \%$, and accuracy $-62.67 \%$. There was a statistically significant positive relationship between the presence of the criterion and SLE (association coefficient of 0.71). We found that renal disorders were 6.00 times more prevalent in patients with SLE than in patients with other rheumatic diseases.

Neurologic disorders, including seizures and/or mental disorders, were detected in 46 patients (12.43\%) of the SLE group. Only three patients $(1.30 \%)$ of the comparison group had neurologic disorders. The sensitivity of the criterion was $12.43 \%$, specificity $-98.70 \%$, accuracy $-45.60 \%$. There 
was a statistically significant positive relationship between the presence of the criterion and SLE (association coefficient of 0.83 ). We found that neurologic disorders were 10.79 times more prevalent in patients with SLE than in patients with other rheumatic diseases.

Hematologic disorders include hemolytic anemia with reticulocytosis, thrombocytopenia, lymphopenia, and/or leukopenia. According to the results of laboratory examinations, we detected hematological disorders in 169 patients (45.68\%) of the SLE group and in 111 patients (47.44\%) of the comparison group. According to our calculations, the presence of any hematological disorder does not indicate SLE - there was no statistically significant relationship between the presence of the criterion and SLE (sensitivity was $45.68 \%$, specificity $-52.56 \%$, accuracy $-48.34 \%$, association coefficient of -0.04). According to the results of statistical analysis, hematological disorders are less prevalent in patients with SLE than in patients with other rheumatic diseases.

Immunologic disorders (anti-dsDNA antibodies, antiphospholipid antibodies ( $\operatorname{IgM}$ and $\operatorname{IgG}$ ), lupus anticoagulants) were detected in 302 patients $(88.30 \%)$ of the SLE group. Immunologic disorders were also detected in three patients $(3.45 \%)$ of the comparison group. The sensitivity of the criterion was $88.30 \%$, specificity $-96.55 \%$, accuracy $-89.98 \%$. There was a statistically significant positive relationship between the criterion and SLE (association coefficient of 0.99). Immunologic disorders were 211.40 times more prevalent in patients with SLE than in patients with other rheumatic diseases.

ANAs were detected in 249 patients (87.99\%) of the SLE group and in nine patients (15.79\%) of the comparison group. The sensitivity of ANAs as the criterion for SLE was $87.99 \%$, specificity was $84.21 \%$, and accuracy was $87.35 \%$. There was a statistically significant positive relationship between the presence of the criterion and SLE (association coefficient of 0.95). According to the results of statistical analysis, ANAs were 39.06 times more prevalent in patients with SLE than in patients with other rheumatic diseases.

The analysis of the diagnostic value of the ACR criteria in the case of the SLE group suggests that SLE may be predicted only by some of the currently approved criteria - namely, butterfly rash, photosensitivity, serositis, renal disorders, neurologic disorders, immunologic disorders, and ANAs. Other criteria, such as discoid rash, oral ulcers, hematological disorders, do not help to predict the presence of SLE, and arthritis - on the contrary - rules out the disease.

The results of the second step of the study are shown in Table 2 and in Figure 2. The purpose of the second step was to determine the diagnostic value of the individual components of certain ACR criteria - namely, the presence of pericarditis, pleuritis, proteinuria, cylindruria, hemolytic anemia with reticulocytosis, thrombocytopenia, lymphopenia, leukopenia, anti-dsDNA antibodies, antiphospholipid antibodies (IgM and $\operatorname{IgG}$ ), and lupus anticoagulants.

Table 2. Diagnostic Value of the Components of Certain ACR Criteria for the Classification of Systemic Lupus Erythematosus

\begin{tabular}{|c|c|c|c|c|c|c|c|c|}
\hline No. & \multicolumn{2}{|c|}{ Diagnostic criteria } & Sensitivity, & $\%$ Specificity, \% & Accuracy, \% & $\begin{array}{l}\text { Odds } \\
\text { ratio }\end{array}$ & $\begin{array}{c}\text { Coefficient } \\
\text { of } \\
\text { association }\end{array}$ & $\begin{array}{l}\text { Coefficient } \\
\text { of } \\
\text { contingency }\end{array}$ \\
\hline 1. & \multirow{2}{*}{ Serositis } & Pericarditis & 17.30 & 100.00 & 49.34 & - & 1 & 0.27 \\
\hline 2. & & Pleuritis & 13.51 & 100.00 & 47.02 & - & 1 & 0.24 \\
\hline 3. & \multirow{2}{*}{$\begin{array}{c}\text { Renal } \\
\text { disorders }\end{array}$} & Proteinuria & 46.49 & 85.13 & 60.98 & 4.98 & $0.67 *$ & - \\
\hline 4. & & Cylindruria & 11.35 & 98.63 & 43.80 & 9.22 & $0.80 *$ & - \\
\hline 5. & \multirow{2}{*}{$\begin{array}{c}\text { Neurologic } \\
\text { disorders }\end{array}$} & Seizures & 0.81 & 98.72 & 38.74 & 0.63 & -0.23 & - \\
\hline 6. & & Psychosis & 6.49 & 100.00 & 42.71 & - & 1 & 0.16 \\
\hline 7. & \multirow{4}{*}{$\begin{array}{c}\text { Hematologic } \\
\text { disorders }\end{array}$} & $\begin{array}{l}\text { Hemolytic anemia } \\
\text { with reticulocytosis }\end{array}$ & 02.16 & 83.33 & 33.61 & 0.11 & -0.80 & - \\
\hline 8. & & Thrombocytopenia & 13.24 & 97.44 & 45.86 & 5.80 & $0.71 *$ & - \\
\hline 9. & & Lymphopenia & 07.57 & 69.23 & 31.46 & 0.18 & -0.69 & - \\
\hline 10. & & Leukopenia & 37.30 & 83.33 & 55.13 & 2.97 & 0.49 & - \\
\hline 11. & \multirow{4}{*}{$\begin{array}{l}\text { Immunologic } \\
\text { disorders }\end{array}$} & Anti-dsDNA & 90.19 & 96.29 & 91.47 & 239.20 & $0.99 *$ & - \\
\hline 12. & & $\begin{array}{c}\text { Antiphospholipid } \\
\text { IgM }\end{array}$ & 26.95 & 100.00 & 28.47 & - & 1 & 0.09 \\
\hline 13. & & $\begin{array}{c}\text { Antiphospholipid } \\
\text { IgG }\end{array}$ & 24.00 & 100.00 & 25.49 & - & 1 & 0.08 \\
\hline 14. & & Lupus anticoagulant & 3.39 & - & 03.39 & - & - & - \\
\hline
\end{tabular}

Note: $*-$ the presence of SLE is statistically significant, $\mathrm{p}<0.05$. 


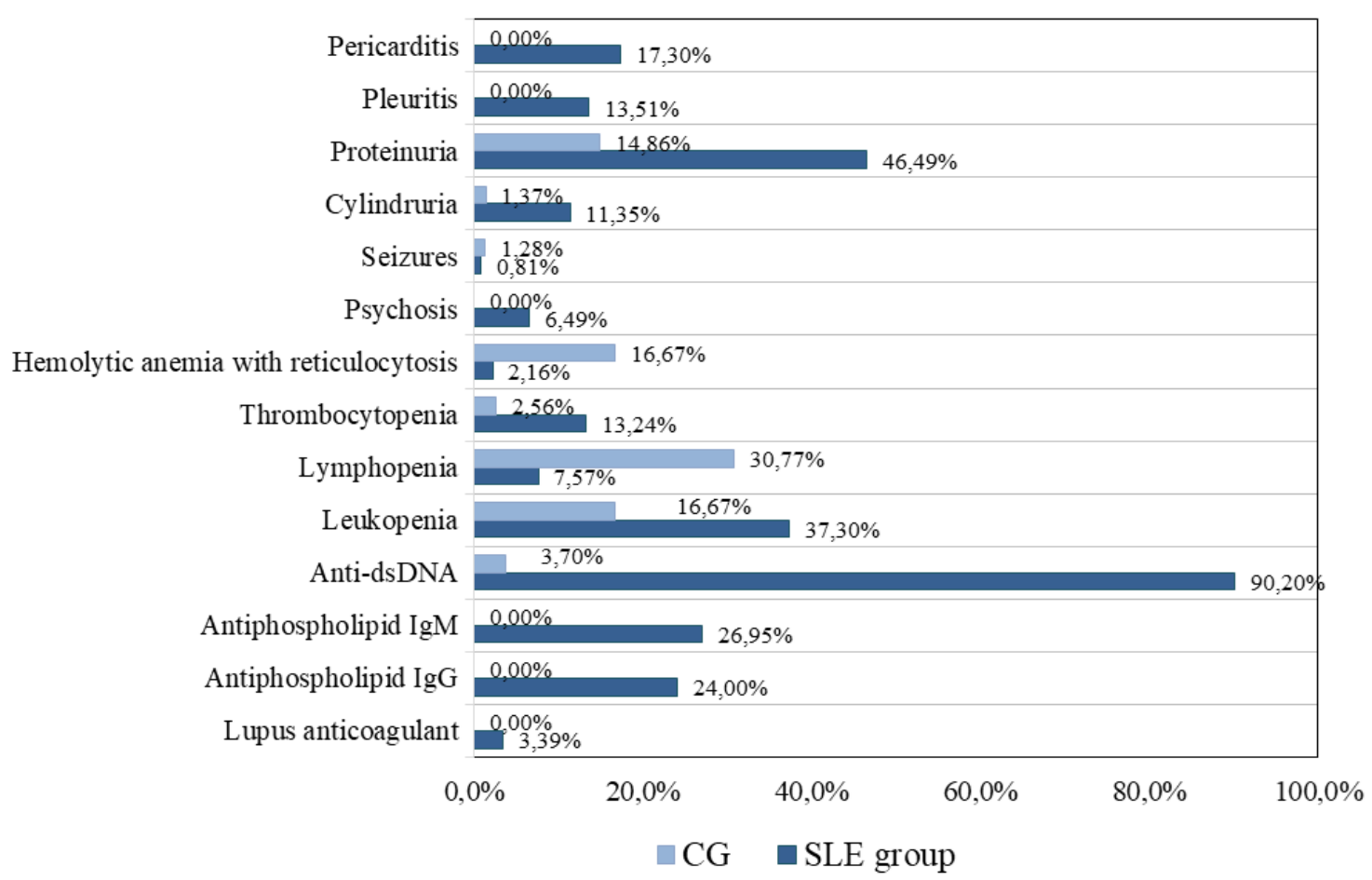

Fig.2. Prevalence of the components of certain ACR criteria in patients with SLE and in patients with other rheumatic diseases

Pericarditis was detected in 64 patients (17.30\%) of the SLE group, and pleuritis was detected in 50 patients $(13.51 \%)$ of the SLE group. Neither pericarditis nor pleuritis was detected in patients $(0.00 \%)$ of the comparison group. The sensitivity of the pericarditis component was $17.30 \%$, specificity $-100.00 \%$, accuracy $-49.34 \%$. There was no statistically significant relationship between the presence of pericarditis and SLE (contingency coefficient of 0.27 ). The sensitivity of the pleuritis component was $13.51 \%$, specificity $-100.00 \%$, accuracy $-47.02 \%$. There was no statistically significant relationship between the presence of pleuritis and SLE (contingency coefficient of 0.24).

Proteinuria was detected in 172 patients $(46.49 \%)$ of the SLE group and in 33 patients (14.86\%) of the comparison group. Cylindruria occurred in 42 patients (11.35\%) of the SLE group and in three patients $(1.37 \%)$ of the comparison group. The sensitivity of the proteinuria component was $46.49 \%$, specificity $-85.14 \%$, accuracy $-60.98 \%$. There was a statistically significant positive relationship between the presence of proteinuria and the diagnosis of SLE (association coefficient of 0.67). The sensitivity of the cylindruria component was $11.35 \%$, specificity $-98.63 \%$, accuracy $43.80 \%$. There was also a statistically significant positive relationship between the presence of cylindruria and the diagnosis of SLE (association coefficient of 0.80 ).

Seizures and psychosis were found in the medical records of three patients $(0.81 \%)$ and 24 patients $(6.49 \%)$ of the SLE group, respectively. Three patients $(1.28 \%)$ of the comparison group had seizures, and nobody $(0.00 \%)$ had psychosis. There were no statistically significant relationships between the presence of seizures and SLE (sensitivity $0.81 \%$, specificity $98.72 \%$, accuracy $38.74 \%$, association coefficient of -0.23 ), as well as between the presence of psychosis and SLE (sensitivity $6.49 \%$, specificity $100.00 \%$, accuracy $42.72 \%$, contingency coefficient of 0.16 ).

According to the results of examinations, we detected following hematologic disorders in the patients of the SLE group: hemolytic anemia - in eight patients (2.16\%), thrombocytopenia - in 49 patients (13.24\%), lymphopenia - in 28 patients (7.57\%) and leukopenia - in 138 patients $(37.30 \%)$. 39 patients $(16.67 \%)$ of the comparison group had anemia, six patients $(2.56 \%)$ of the comparison group had thrombocytopenia, 72 patients $(30.77 \%)$ of the comparison group had lymphopenia, and 39 patients (16.67\%) of the comparison group had leukopenia. There was a statistically significant positive relationship between the presence of thrombocytopenia and SLE (sensitivity 13.24\%, specificity $97.44 \%$, accuracy $45.86 \%$, association coefficient of 0.71 ). The relationship between the 
presence of anemia and SLE was statistically significant and negative (sensitivity $2.16 \%$, specificity $83.33 \%$, accuracy $33.61 \%$, association coefficient of -0.80 ), i.e. the prevalence of anemia was significantly lower in patients with SLE than in patients with other rheumatic diseases. Lymphopenia was also significantly more prevalent in patients with other rheumatic diseases than in patients with SLE (sensitivity $7.57 \%$, specificity $69.23 \%$, accuracy $31.46 \%$, association coefficient of -0.69 ). There was no statistically significant relationship between the presence of leukopenia and SLE (sensitivity $37.30 \%$, specificity $83.33 \%$, accuracy $55.13 \%$, association coefficient of 0.49 ).

Anti-dsDNA antibodies were detected in 276 patients $(90.20 \%)$ of the SLE group. Antiphospholipid antibodies $\operatorname{IgM}$ were detected in 38 patients $(26.95 \%)$, and antiphospholipid antibodies IgG were detected in 36 patients $(24.00 \%)$ of the SLE group. Two patients $(3.39 \%)$ of the SLE group had lupus anticoagulants. Only three patients (3.70\%) of the comparison group had antidsDNA antibodies. No other immunologic disorders were detected in patients of the comparison group. We found a statistically significant positive relationship between the presence of anti-dsDNA antibodies and SLE (sensitivity $90.20 \%$, specificity $96.30 \%$, accuracy $91.47 \%$, association coefficient of 0.99). There were no statistically significant relationships between the presence of other immunologic disorders and SLE: between the presence of antiphospholipid antibodies IgM and SLE (sensitivity $29.95 \%$, specificity $100.00 \%$, accuracy $28.47 \%$, contingency coefficient of 0.08 ), antiphospholipid antibodies IgG and SLE (sensitivity $24.00 \%$, specificity $100.00 \%$, accuracy $25.49 \%$, contingency coefficient of 0.07), lupus anticoagulants and SLE (sensitivity $3.40 \%$ ).

Therefore, we identified proteinuria, cylindruria, thrombocytopenia, and the presence of antidsDNA antibodies as independent statistically significant criteria for the diagnosis of SLE, with high sensitivity, specificity, and accuracy. Other criteria (disorders), such as hemolytic anemia and lymphopenia, in contrast, are more prevalent in patients with other rheumatic diseases than in patients with SLE and are more likely to predict the absence of the latter. Pericarditis, pleuritis, seizures, psychosis, leukopenia, the presence of antiphospholipid antibodies IgM and IgG, and lupus anticoagulants have no statistically significant relationship with the disease.

Conclusions. We determined the diagnostic value of the criteria for the classification of systemic lupus erythematosus proposed by the American College of Rheumatology and found that butterfly rash, photosensitivity, serositis (pleuritis or pericarditis), neurologic disorders (seizures or psychosis), thrombocytopenia, renal disorders (proteinuria or cylindruria), anti-dsDNA and antinuclear antibodies had statistically significant sensitivity, specificity, and accuracy. In contrast, other ACR criteria were more characteristic of a number of other rheumatic diseases.

\section{REFERENCES}

1. Anders Album, Stefan Norrell. Introduction to Modern Epidemiology. Maty Rahu; trans. English. I. Bonya. Tallinn: Inst Experim. and klin. Medicine (Estonia), Dat. protyvorak. o-vo, 1996. 122 p.

2. Cohen AS, Canoso JJ. Criteria for the classification of systemic lupus erythematosus-status 1972. Arthritis Rheum. 1972; 15(5):540-543.

3. Cohen AS, Reynolds WE, Franklin EC et al. Preliminary criteria for the classification of systemic lupus erythematosus. Bull Rheum Dis. 1971; 21:643-648.

4. Hochberg MC. Updating the American College of Rheumatology revised criteria for the classification of systemic lupus erythematosus. Arthritis Rheum 1997;40(9):1725.

5. Tan EM, Cohen AS, Fries JF, Masi AT, McShane DJ, Rothfield NF et al. The 1982 revised criteria for the classification of systemic lupus erythematosus. Arthritis Rheum. 1982; 25(11):1271-1277. 